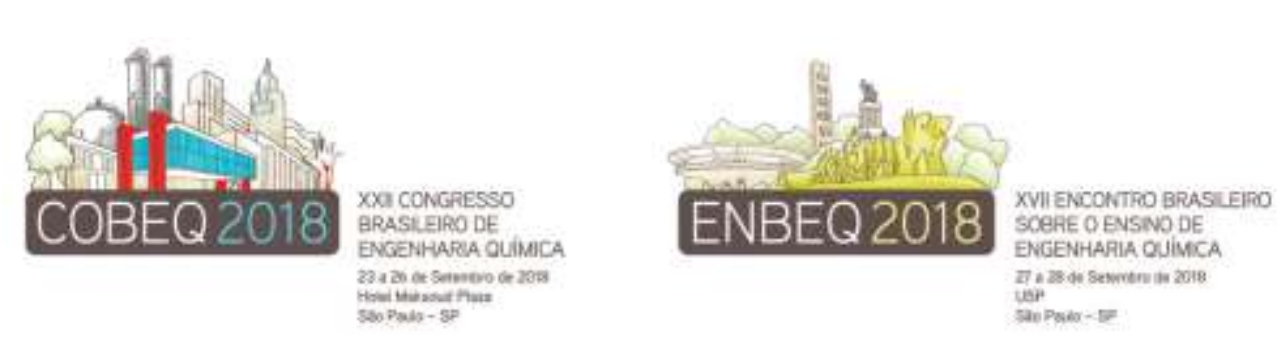

\title{
RETICULAÇÃO DE FILMES POLIMÉRICOS DE GELATINA E AMIDO PELA ADIÇÃO DE GLUTARALDEÍDO: EFEITO DAS DIFERENTES CONDIÇÕES DE SECAGEM
}

\author{
SCOPEL BS ${ }^{1}$, BALDASSO C ${ }^{2}$, DETTMER A ${ }^{3}$ e SANTANA RMC ${ }^{1}$ \\ ${ }^{1}$ Universidade Federal do Rio Grande do Sul, Programa de Pós-Graduação em Engenharia de \\ Minas, Metalúrgica e Materiais \\ ${ }^{2}$ Universidade de Caxias do Sul, Programa de Pós-Graduação em Engenharia de Processos e \\ Tecnologias \\ ${ }^{3}$ Universidade de Passo Fundo, Programa de Pós-Graduação em Ciência e Tecnologia de \\ Alimentos \\ E-mail para contato: bianca.scopel1@gmail.com
}

\begin{abstract}
RESUMO - Neste trabalho, objetivou-se determinar o quanto as condições de processamento de solução filmogênica e de secagem de filmes de amido e gelatina afetam a reticulação deste material pela ação do glutaraldeído. Testou-se o emprego de elevada temperatura $\left(90^{\circ} \mathrm{C}\right)$ ou ausência desta no tratamento da solução filmogênica após adição de glutaraldeído, bem como secagem a $22^{\circ} \mathrm{C}$ (5 dias) e $45^{\circ} \mathrm{C}$ ( 1 dia). Constatou-se que, independentemente da condição testada, há a ocorrência da reticulação do material pelo glutaraldeído, o que fica evidenciado pela redução da solubilidade em água $(20 \mathrm{~h})$ do material de 40\% para 20-27\% e aumento na resistência à tração de 4 MPa para 8-13 MPa. Tem-se assim que o glutaraldeído pode ser empregado na reticulação e melhoria de propriedades de filmes de gelatina e amido, sem restrições de condições de temperatura de processamento e secagem, dentro do aqui testado.
\end{abstract}

\section{INTRODUÇÃO}

Filmes poliméricos de amido e gelatina são comumente estudados para aplicações como embalagens alimentícias (Acosta et al., 2015; Al-Hassan e Norziah, 2017). Há também estudos para seu uso em sistemas de liberação de drogas (Garcia et al., 2017) e na agricultura, como cobertura de solo (Scopel et al., 2017). Entretanto, de acordo com Paoli (2008), polímeros como os formados por amido e gelatina são caracterizados por serem hidrobiodegradáveis. Nestes, a hidrólise da molécula polimérica leva à formação de moléculas menores usadas como nutrientes por microrganismos, o que implica na utilização destes polímeros apenas em aplicações em que não haja contato direto com água, limitando seu uso.

Uma das maneiras de diminuir a interação destes filmes com água é por meio de sua reticulação. Um dos agentes reticulantes de cadeias de amido e gelatina é o glutaraldeído. Além de promover a reticulação das cadeias de amido (Phromsopha \& Baimark, 2014), o glutaraldeído promove reticulação na gelatina por meio de ligações entre seus grupamentos hidroxila e os grupamentos amina da gelatina (Englert et al., 2007). 


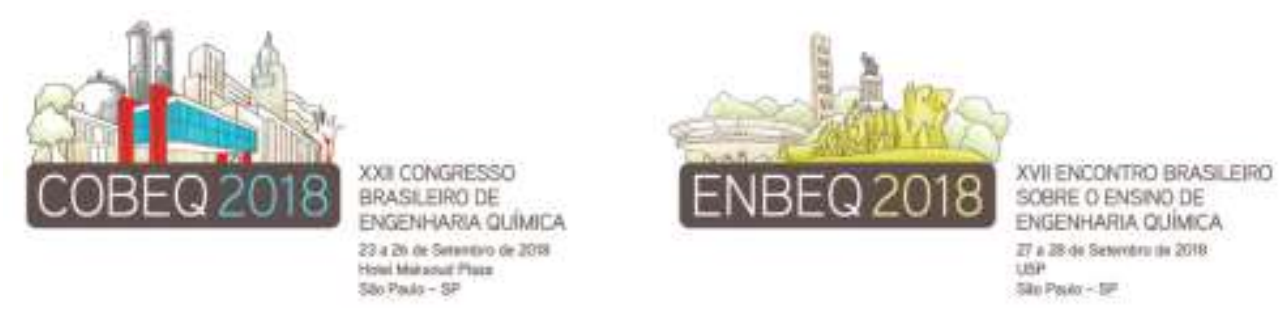

Neste trabalho, diferentes temperaturas de processamento da solução filmogênica e de secagem de filme foram testadas a fim de verificar em quais condições a reticulação, e consequente melhoria de propriedades dos filmes, ocorre.

\section{METODOLOGIA}

Filmes poliméricos de amido e gelatina foram produzidos pela técnica de casting em cinco diferentes condições. Empregou-se formulação com 2,38 g de gelatina comercial, 1,12 $\mathrm{g}$ de amido de milho, 0,91 g de glicerol e, quando empregado, $0,14 \mathrm{~mL}$ de glutaraldeído $50 \%$.

Amido e gelatina foram misturados em água totalizando volume de $105 \mathrm{~mL}$. O sistema foi aquecido, sob agitação, a $90^{\circ} \mathrm{C}$ por $20 \mathrm{~min}$. A solução resultante foi transferida a um béquer contendo o glicerol e resfriada a temperatura ambiente. Em temperatura ambiente $\left(25^{\circ} \mathrm{C}\right)$, foi adicionado o glutaraldeído. Após a adição do glutaraldeído, três soluções passaram pelas condições de tratamento apresentadas na Tabela 1, e outras três não sofreram novos períodos de aquecimento. Por fim, as soluções foram espalhadas em placas de vidro (comprimento 21,0 $\mathrm{cm}$ e largura $13,5 \mathrm{~cm}$ ) e secas conforme condições da Tabela 1.

Tabela 1 - Diferentes condições de processamento de solução filmogênica e secagem de filme testadas para a mistura de gelatina, amido de milho, glicerol e glutaraldeído

\begin{tabular}{cccccc}
\hline Código da Amostra & $\begin{array}{c}\text { Adição de } \\
\text { glutaraldeído? }\end{array}$ & $\begin{array}{c}\text { Tratamento da solução } \\
\text { filmogênica } \\
\text { Tempo } \\
(\min )\end{array}$ & $\begin{array}{c}\text { Temperatura } \\
\left({ }^{\circ} \mathrm{C}\right)\end{array}$ & $\begin{array}{c}\text { Secagem dos Filmes } \\
\text { Tempo } \\
(\text { dias })\end{array}$ & $\begin{array}{c}\text { Temperatura } \\
\left({ }^{\circ} \mathrm{C}\right)\end{array}$ \\
\hline $90 / 22$ & Sim & 20 & 90 & 5 & 22 \\
Sem trat/22 & Sim & 0 & -- & 5 & 22 \\
Sem glut/Sem trat/45 & Não & 0 & -- & 1 & 45 \\
Sem trat/45 & Sim & 0 & -- & 1 & 45 \\
$35 / 45$ & Sim & 20 & 35 & 1 & 45 \\
$90 / 45$ & Sim & 20 & 90 & 1 & 45 \\
\hline
\end{tabular}

Os filmes foram caracterizados com relação a sua espessura utilizando-se um medidor de camadas (Modelo 345, Elcometer). A solubilidade dos filmes foi medida de acordo com a perda de massa das amostras em água após $20 \mathrm{~h}$ de agitação a $25^{\circ} \mathrm{C}$ e $80 \mathrm{rpm}$. Propriedades mecânicas foram medidas de acordo com a norma ASTM D882-2012.

\section{RESULTADOS E DISCUSSÃO}

A Tabela 2 apresenta os resultados de caracterização para os filmes obtidos. As maiores diferenças com relação às propriedades medidas são percebidas na amostra que não recebeu a adição de glutaraldeído (Sem glut/Sem trat/45). Todas as demais condições de produção permitiram redução na solubilidade das amostras, o que indica a eficaz reticulação das cadeias poliméricas. 


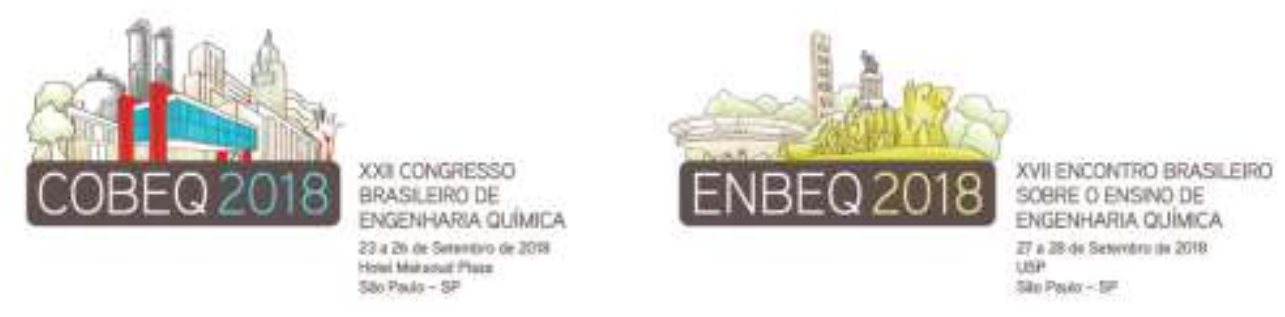

Tabela 2 - Caracterização dos filmes de gelatina, amido de milho, glicerol e glutaraldeído produzidos em diferentes condições de processamento da solução filmogênica e secagem do

\begin{tabular}{ccccc}
\hline Amostra & $\begin{array}{c}\text { Espessura } \\
(\mu \mathrm{m})\end{array}$ & $\begin{array}{c}\text { Solubilidade } \\
(\%)\end{array}$ & $\begin{array}{c}\text { Resistência à } \\
\text { Tração }(\mathrm{MPa})\end{array}$ & $\begin{array}{c}\text { Alongamento na } \\
\text { Ruptura }(\%)\end{array}$ \\
\hline $90 / 22$ & $108 \pm 6$ & $27 \pm 1$ & $13 \pm 3$ & $146 \pm 15$ \\
Sem trat/22 & $112 \pm 4$ & $26 \pm 1$ & $12 \pm 2$ & $162 \pm 24$ \\
Sem glut/Sem trat/45 & $104 \pm 6$ & $40 \pm 1$ & $4 \pm 1$ & $130 \pm 64$ \\
Sem trat/45 & $112 \pm 5$ & $20 \pm 3$ & $10 \pm 4$ & $258 \pm 105$ \\
$35 / 45$ & $100 \pm 4$ & $24 \pm 1$ & $8 \pm 2$ & $189 \pm 44$ \\
$90 / 45$ & $90 \pm 7$ & $25 \pm 1$ & $9 \pm 3$ & $268 \pm 34$ \\
\hline
\end{tabular}

Observa-se maior aumento na resistência à tração das amostras secas a $22^{\circ} \mathrm{C}$ e maior aumento no alongamento na ruptura nas amostras secas a $45^{\circ} \mathrm{C}$ (em especial na Sem trat $/ 45$ e 90/45). As diferenças nas propriedades mecânicas devem estar atreladas a um maior grau de recristalização das cadeias de amido quando secas em menores temperaturas e por maiores tempos (o que aumenta sua resistência à tração), bem como ao fenômeno inverso, reduzindo grau de recristalização das cadeias quando secas a maior temperatura e menor tempo (o que aumenta alongamento na ruptura) (Scopel et al., 2017).

O fenômeno mais interessante dos testes aqui apresentados, entretanto, reside no fato de que, independentemente da condição na qual a solução filmogênica foi produzida e da temperatura/tempo de secagem, as cadeias poliméricas foram reticuladas na presença de glutaraldeído, o que é verificado principalmente, pela redução na solubilidade. Isto indica que, ainda que a reação de reticulação seja acelerada pela temperatura (o que foi verificado em laboratório), ela não deixa de ocorrer na ausência de aplicação de temperaturas elevadas.

A Figura 1 apresenta figuras das amostras Sem glut/Sem trat/45, Sem trat/22 e Sem trat/45. Prova-se o discutido no parágrafo anterior pela coloração da amostra Sem trat/22 (amarela, assim como da Sem trat/45). A coloração amarelada advém da reação entre glutaraldeído e grupamentos lisina e hidroxilisina da gelatina, que originam uma base de Schiff, de cor amarelada (Tian et al., 2016). A amostra Sem trat/22 não passou por qualquer tratamento térmico após adição de glutaraldeído (a solução filmogênica não foi aquecida nem a $35^{\circ} \mathrm{C}$ nem a $90^{\circ} \mathrm{C}$ e a secagem do filme ocorreu a $22^{\circ} \mathrm{C}$ ). Mesmo assim, ela apresenta a coloração amarelada indicativa da reticulação das cadeias proteicas pela ação do glutaraldeído.

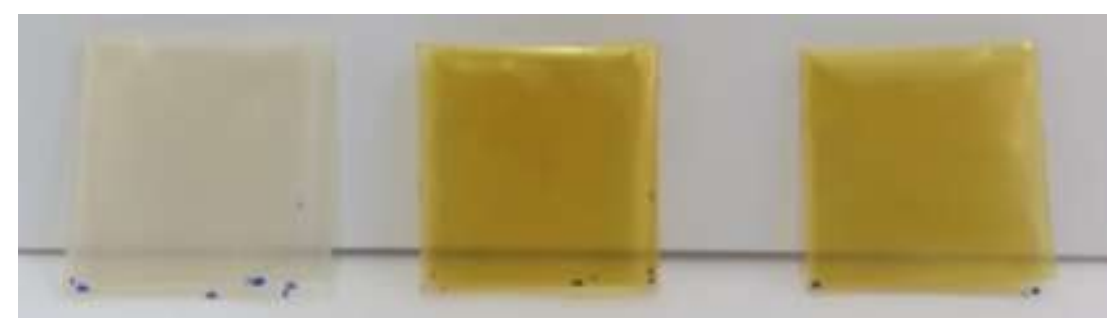

Figura 1 - Amostras Sem glut/Sem trat/45 (esquerda), Sem trat/22 (centro) e Sem trat/45 (direita). 


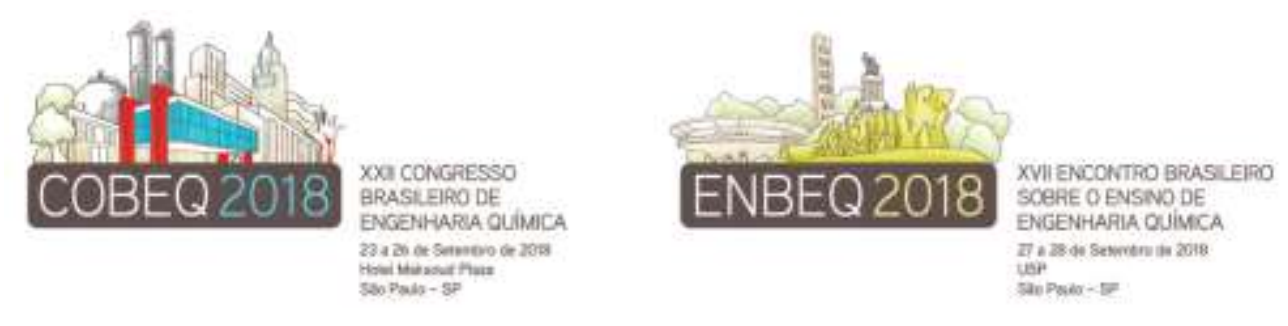

\section{CONCLUSÃO}

A partir dos resultados apresentados neste trabalho, verifica-se que o glutaraldeído pode ser empregado como agente reticulante de filmes de amido e gelatina plastificados com glicerol. Ele promove redução da solubilidade dos filmes em água - $20 \mathrm{~h}$ (que varia de $40 \%$ sem a adição de glutaraldeído para 20 a $27 \%$ com sua adição) e aumento na resistência à tração (de $4 \mathrm{MPa}$ sem a adição de glutaraldeído para 8 a $13 \mathrm{MPa}$ com sua adição). Em todas as condições de processamento da solução filmogênica pós adição de glutaraldeído $\left(90^{\circ} \mathrm{C}\right.$ e sem tratamento térmico) e de secagem $\left(22^{\circ} \mathrm{C}-5\right.$ dias e $45^{\circ} \mathrm{C}-1$ dia), houve melhora nas propriedades dos filmes. Com isso, conclui-se que a produção de filmes de amido e gelatina reticulados com glutaraldeído pode ser realizada sem restrições nas condições de produção, dentro do aqui testado, adaptando-se, portanto, às condições de produção e secagem disponíveis no ambiente de produção.

\section{REFERÊNCIAS}

ACOSTA S, CHIRALT A, SANTAMARIANA P, GONZÁLEZ-MARTINEZ C, CHÁFER M, Antifungal films based on starch-gelatin blend, containing essential oils. Food Hydrocoll., v. 61, p. 233-240, 2016.

AL-HASSAN AA, NORZIAH MH, Starch-gelatin edible films: Water vapor permeability and mechanical properties as affected by plasticizers. Food Hydrocoll., v. 26, p. 108-117, 2012.

ENGLERT C, BLUNK T, MULLER R, SCHULZE VON GLASSER S, BAUMER J, FIERLBECK J, HEID I, NERLICH M, HAMMER J, Bonding of articular cartilage using a combination of biochemical degradation and surface cross-linking. Arthritis Res Ther, v. 9, p. 47, 2007.

GARCIA VADS, BORGES JG, MAZALLI MR, LAPA-GUIMARÃES JG, VANIN FM, CARVALHO RA, Gelatin and pregelatinized starch orally disintegrating films: Properties and stability of vitamin C. J Appl Polym Sci., v. 134, 2017.

PAOLI MAD. Degradalção e estabilização de polímeros. São Paulo: Chemkeys, 2008.

PHROMSOPHA T, BAIMARK Y, Preparation of Starch/Gelatin Blend Microparticles by a Water-in-Oil Emulsion Method for Controlled Release Drug Delivery. Int J Biomater, v. 2014, $6 p, 2014$.

SCOPEL BS, RIBEIRO ME, DETTMER A, BALDASSO C. Cornstarch-Gelatin Films: Commercial Gelatin Versus Chromed Leather Waste Gelatin and Evaluation of Drying Conditions. J. Polym. Environ., p. 1-9, 2017.

ZHENHUA T, WENTAO L, GUOYING L, The microstructure and stability of collagen hydrogel cross-linked by glutaraldehyde. Polym. Degrad. Stab., V. 130, P. 264-270, 2016. 